\title{
Antibacterial and Microstructure Properties of Titanium Surfaces Modified with Ag-Incorporated Nanotube Arrays
}

\author{
Guangzhong Li $i^{a}$,Quan-ming Zhao ${ }^{b^{*}}$, Hui-lin Yangc, Li Cheng ${ }^{a}$ \\ ${ }^{a}$ State Key Laboratory of Porous Metal Materials, Northwest Institute for Nonferrous Metal Research, \\ Xi'an 710016, China. \\ ${ }^{b}$ Department of Orthopaedics, Wuxi People's Hospital, Nanjing Medical University, \\ Wuxi City 214023, Jiangsu Province, P.R. China. \\ ${ }^{c}$ Department of Orthopaedics, The First Affiliated Hospital of Soochow University, \\ Suzhou 215006, China.
}

Received: September 7, 2015; Revised: March 2, 2016; Accepted: April 15, 2016

\begin{abstract}
Although titanium (Ti) and its alloys have been widely used as implants in clinical settings, failures still occur mainly due to poor bioactivity and implant-associated infections. Here, we coated Ti implants with $\mathrm{TiO}_{2}$ nanotubes (TNTs) incorporated with the antibacterial agent Ag to produce Ag-TNTs, through anodization in $\mathrm{AgNO}_{3}$ and xenon light irradiation. We characterized surface morphology and composition of the coating with scanning electron microscopy (SEM) and X-ray diffraction (XRD), respectively. We investigated surface topography of the coatings by atomic force microscopy (AFM) operated in the tapping mode. The results indicate that Ag was successfully doped onto the TNTs, and that the nanoparticles were mainly distributed on the surface of TNTs. Finally, our antibacterial experiments reveal that Ag-TNTs on Ti implants exhibit excellent antibacterial activities, which promises to have significant clinical applications as implants.
\end{abstract}

Keywords: Anodization, Titanium, Ag nanoparticles, Antibacterial activities

\section{Introduction}

Titanium (Ti) and its alloy are used in orthopedic implant applications due to their excellent wear, corrosion resistance and acceptable biocompatibility properties ${ }^{1}$. However, lacking in biological activity means they tend to be encapsulated by fibrous tissue, which results in low level chemical bonding with the bone ${ }^{2}$. Moreover, the interface bonding between the Ti implant and bone tissue has a tendency to loosen over time due to the poor osseointegration. Another prominent reason for Ti implant failure is implant-associated infections ${ }^{3}$. On one hand, surgical trauma reduces the host's resistance to fight infections, while on the other, the implant itself as foreign body increases the risk of infection, resulting in propagation and invasion of bacteria.

Once infection occurs, conventional antibiotic therapy is generally ineffective, which can lead to implant removal, causing great pain and distress to the patient. Furthermore, studies have confirmed that once the bacteria are isolated ${ }^{4}$, systemic treatment of antibiotics do not deliver effective drug concentration levels locally at the implant site. Once the pathogen is attached to the bone or implants surface, it will generate metabolic and phenotypic changes, which result in pathogens producing resistance and escaping immune surveillance ${ }^{5}$. Lower levels of antibiotics may also become the driving force of bacterial drug resistance ${ }^{6}$. Hence, the prevention and control of implant-associated infections has attracted much attention in recent years.

*e-mail: abc8385@163.com
Surface modification of implant materials is an effective way to reduce or inhibit the occurrence of implant-associated infections. Implants modified with antibacterial agents will not only ensure a higher concentration of antibacterial activity delivered on the surface of the implant, but will also avoid the many potential problems caused by the excessive use of antibiotics, such as bacterial drug resistance and the formation of super bacteria. Various surface modification techniques have been used to introduce antibacterial agents to the surface of implants, including sol-gel ${ }^{7}$, plasma spray $^{8}$ and anodic oxidation $^{9}$. Among these methods, anodic oxidation is the most versatile and low costing technique. It can form ordered and uniform TNT arrays, and the morphology, pore size, length and wall thickness of the nanotubes can be accurately controlled by changing the composition of the electrolyte, $\mathrm{pH}$ and time. TNTs have good biocompatibility due to their low elastic modulus, similar to that of natural bone tissue ${ }^{10}$. More importantly, the nanoscale tubular topography means that implants exhibit distinct biological properties, such as promoting osteogenesis and providing antibacterial action by carrying biological activation factors and agents ${ }^{11}$.

Recently, antibacterial agents including silver (Ag), copper $(\mathrm{Cu})$ and zinc $(\mathrm{Zn})$ incorporated onto TNTs have attracted much attention ${ }^{12-14}$. Among those, the incorporation of $\mathrm{Ag}$ has gained particular attention due to its broad-spectrum of antibacterial properties and high-security. Studies on the antibacterial mechanisms of Ag have shown that it binds to the bacterial cell wall, damaging the function of the bacterial 
cell membrane ${ }^{15}$. Ag also inhibits the proliferation of bacteria through interactions with the DNA structure of the bacteria ${ }^{16}$.

In this study, we coated TNTs incorporated with the antibacterial agent Ag to produce Ag-TNTs, through anodization in $\mathrm{AgNO}_{3}$ and xenon light irradiation. We fully characterized the morphology, microstructure, chemical composition and chemical state of the coatings. We also examined the antibacterial activity of the coatings against Staphylococcus aureus (S. aureus).

\section{Material and methods}

\subsection{Sample preparation}

We polished commercially pure Ti plates (10 $\mathrm{mm} \times 5 \mathrm{~mm} \times 0.5 \mathrm{~mm}$ ) and ultrasonically cleaned them in acetone, ethanol and deionized water. We then eroded them in $\mathrm{HF}-\mathrm{HNO}_{3}-\mathrm{H}_{2} \mathrm{O}$ solution, cleaned them in deionized water and dried them with a $\mathrm{N}_{2}$ stream. We fabricated the vertically aligned $\mathrm{TiO}_{2}$ nanotube arrays in an electrolyte of $1 \mathrm{vol} \%$ $\mathrm{HF}, 2.0 \mathrm{vol} \% \mathrm{HNO}_{3}$ at $20 \mathrm{~V}$ for $2 \mathrm{~h}$; we used a platinum electrode as the cathode. After being anodized, we rinsed the samples with deionized water and dried with a $\mathrm{N}_{2}$ stream.

We prepared $1 \mathrm{~mol} / \mathrm{L} \mathrm{AgNO}_{3}$ solution by dissolving a certain amount of $\mathrm{AgNO}_{3}$ into deionized water. We next immersed the prepared $\mathrm{TiO}_{2}$ nanotube samples in the solution for $30 \mathrm{~min}$ and washed them in deionized water before leaving them to dry. Sunlight was simulated with a $300 \mathrm{~W}$ xenon arc lamp with an AM 1.5 G filter. The light intensity was set using a NREL calibrated crystalline silicon solar cell. After irradiation using the xenon lamp for $2 \mathrm{~h}, \mathrm{Ag}$ ions were reduced to $\mathrm{Ag}$ nanoparticles.

\subsection{Surface characterization}

We employed field-emission scanning electron microscopy (SEM) and atomic force microscopy (AFM) to evaluate surface morphology. We measured the elemental composition and distribution of the sample using energy-dispersive X-ray spectrometer (EDS). We then characterized the crystal structures of Ag-TNTs using X-ray diffraction spectrometer (XRD).

\subsection{Evaluation of antibacterial activity}

We selected $S$. aureus, which is gram-positive, for the antibacterial activity test. We adjusted the initial concentration of $S$. aureus bacteria to $1.0 \times 10^{4} \mathrm{CFU} / \mathrm{mL}$ by dilution. Then, we dropped $100 \mu \mathrm{L}$ of bacterial solution onto each specimen, and a polyethylene film was placed over the treated specimen. After incubation at $37^{\circ} \mathrm{C}$ for $24 \mathrm{~h}$, each specimen was transferred to $1 \mathrm{~mL}$ of fresh nutrient broth. We collected the bacterial solution and diluted it with fresh nutrient broth by a factor of $10^{4}$. Next, we plated $100 \mu \mathrm{L}$ of diluted nutrient broth onto standard Bacto-Agar culture medium. We incubated the agar plates for $24 \mathrm{~h}$ at $37^{\circ} \mathrm{C}$, counted $S$. aureus CFUs and photographed the plates.

\section{Results and discussion}

\subsection{Surface characterization}

We applied SEM to characterize the surface morphologies of the samples. Fig. 1 (a-c) shows TNTs coated in the absence of Ag doping. At a lower magnification of 50kX (Fig.1a), the nanotubes are densely populated and exhibit a vertically aligned columnar structure. At a higher magnification of
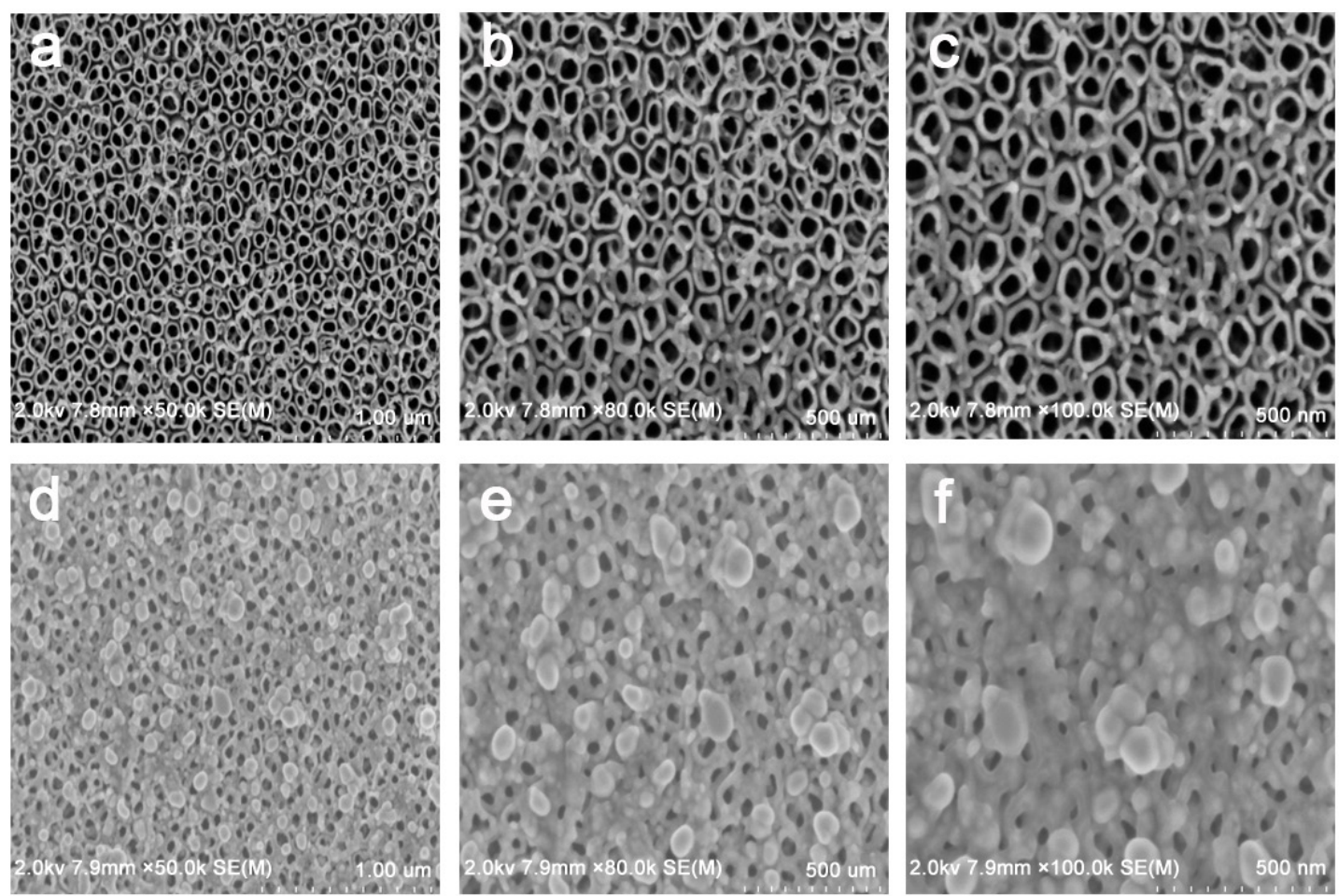

Fig. 1. Surface morphology of TNTs (a, b and c) and Ag-TNT coatings (d, e and f). a and d: 50kX; b and e: 80kX; c and f: 100kX. 
80kX and 100kX (Fig.1b and c), the nanotube arrays follow an independently ordered structure. Typical nanotubes have a diameter of 80-120 nm, and the spacing between adjacent nanotubes is obvious.

Fig.1 (d-f) show coatings containing TNTs incorporated with Ag. At a lower magnification of 50kX (Fig.1d), we observed a large number of nanoparticles unevenly distributed on the surface of the $\mathrm{TiO}_{2}$ nanotubes. At a higher magnification of $80 \mathrm{kX}$ and $100 \mathrm{kX}$ (Fig.1e-f), the nanoparticles vary in size and some of the nanotubes are blocked. Distribution of Ag particles has clearly made the surface appear rougher.

Researchers are keen to apply surface modification technology to prepare coatings on implant surfaces with excellent morphology and good biological activity, realizing integration of structure and biological function. The dual purpose of the mechanical bearing capacity and the biological function is achieved by introducing the biological active elements into the specific structure and the continuous dissolution of the metal elements. The integration of structural and biomedical function is a very challenging and innovative idea, and promises to have important clinic applications.

TNTs can be synthesized by a variety of techniques. However, through adjustments in parameters such as $\mathrm{pH}$, applied voltage and time of anodization, fabrication by anodic oxidation has shown to demonstrate superior control over nanotube dimensions ${ }^{17}$. Moreover, osseointegration is closely related to surface properties of Ti implants, which include surface morphology, roughness, wettability and surface chemical composition of the Ti implant. Surface morphology of TNTs includes micro-, nano- and a mixture of micro-nano structures. Studies have shown that TNT nano- structures are closer to both natural bone tissue chemical properties and morphology, which provides an ideal growth environment for bone regeneration ${ }^{18}$. TNT arrays are a new-type of nanoscale morphology that can regulate cell metabolism, and therefore, became the focus of this research project.

Previous studies have also shown that better osseointegration is achieved through increasing surface roughness ${ }^{19}$. Hence, we employed AFM to obtain detailed morphology of the coatings. Fig. 2 shows the surface topography of the Ag-TNTs at a scale of $3 \mathrm{~nm} \times 3 \mathrm{~nm}$. The results demonstrate a rough surface with some nanoparticles after incorporation of the Ag coating onto the TNTs.

\subsection{EDS analyses of Ag-TNTS}

Figure 3 shows the EDS element mapping of the Ag-TNTs. Figure 3 (a-b) depict a large number of nanoparticles distributed on the surface of the coating. Figure 3(c) shows that 4 wt. $\%$ Ag, 2 wt.\% O and 94\% wt.\% Ti were detected. Figure $3(\mathrm{~g})$ demonstrates that the Ag-TNTs clearly consist of $\mathrm{O}, \mathrm{Ti}$, and $\mathrm{Ag}$, indicating successful doping of Ag on the surface of the Ag-TNTs. Also, the peak height of Ti is high and steep, which indicates that the main component of the surface of the coating remains Ti. Figure 3(d-f) show O, $\mathrm{Ag}$ and Ti element mapping of the coatings respectively. These elements are relatively uniformly distributed on the surface of the coating.

In order to improve the antibacterial properties of $\mathrm{Ti}$ implants, researchers introduce Ag onto TNTs through a variety of ways. However, at certain doses Ag is toxic. Studies have shown that the concentration of $\mathrm{Ag}$ ions and the size of the Ag nanoparticles play a large role in determining its toxicity. For example, $\mathrm{Ag}^{+}$may promote generation of reactive oxygen species and damage to cells, whereas damage from metallic Ag is less prominent, suggesting that the ion state of Ag is the main toxic state ${ }^{20}$. However, high concentrations of Ag nanoparticles can cause cell toxicity ${ }^{21}$. In our study, the "half opening" structure of the TNTs release $\mathrm{Ag}^{+}$slowly, which overcomes the toxic effect from high concentrations of $\mathrm{Ag}^{+}$in the cell.

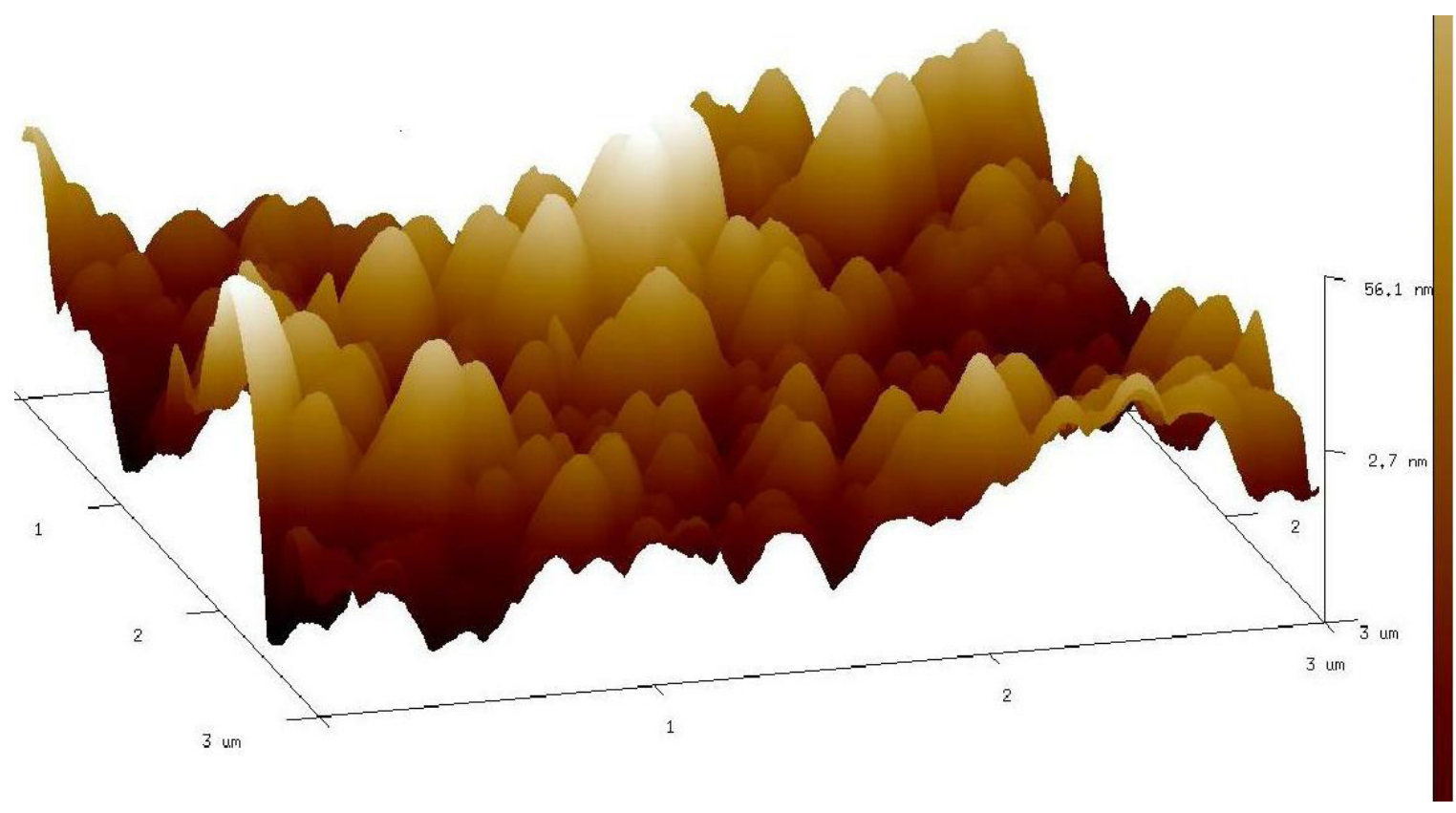

Fig. 2. The 3D AFM image of the surface of Ag-TNT coatings. 

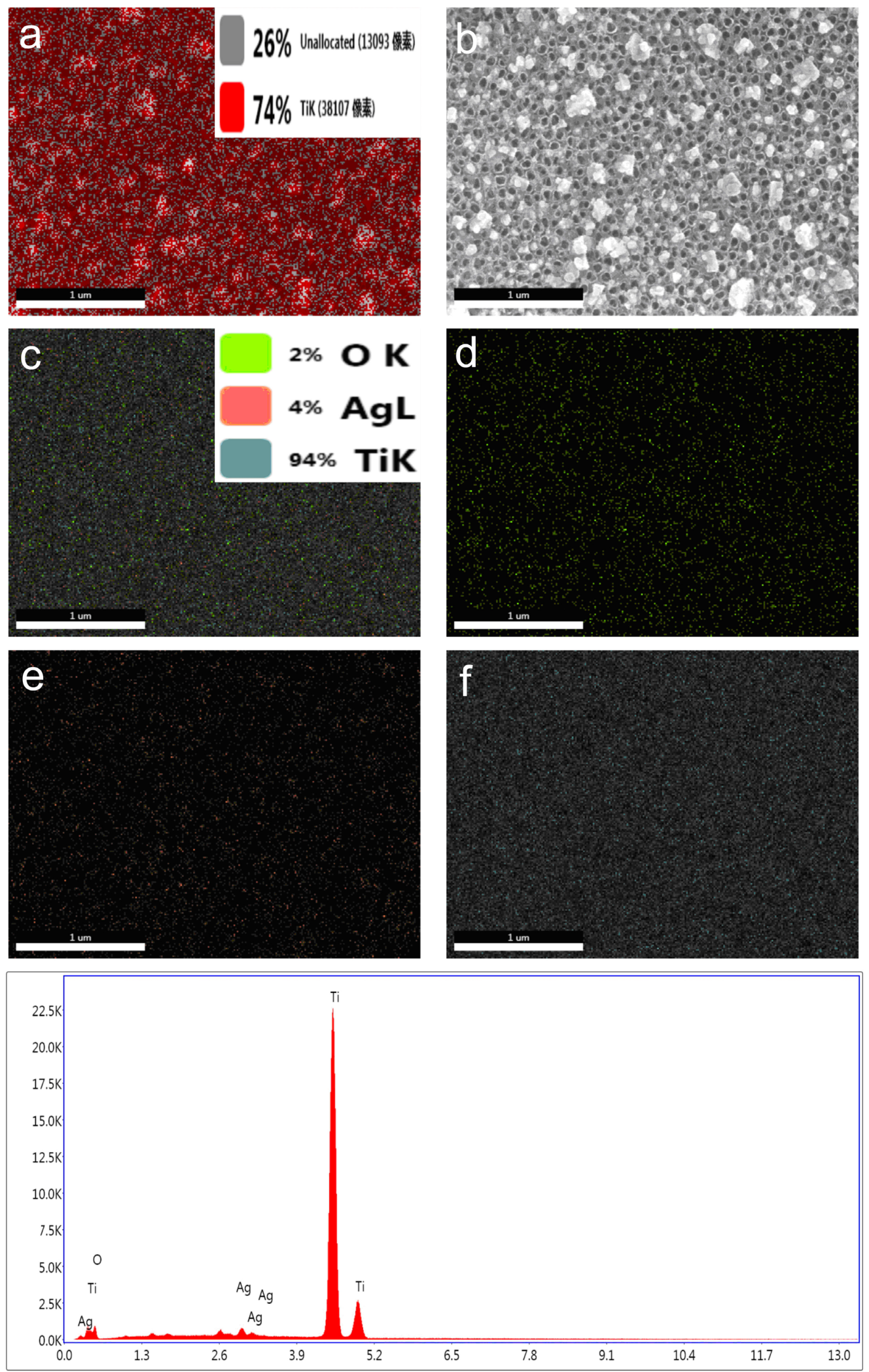

Fig. 3. EDS mapping images and line scan curves. (a and b are surface morphology of Ag-TNT coatings, c represents mixed maps and $\mathrm{d}, \mathrm{e}, \mathrm{f}$ represent $\mathrm{O}, \mathrm{Ag}$ and $\mathrm{f}$ Ti respectively) 


\subsection{XRD patterns of the Ag-TNTs}

Fig. 4 shows the typical XRD patterns of the Ag-TNTs. Obviously, Ti shows strong and sharp peaks. We did not observe any $\mathrm{TiO}_{2}$ diffraction peaks, which may be related to length of the nanotube being too short. Nonetheless, weak Ag diffraction peaks can be observed, which is consistent with EDS analysis.

\subsection{Antibacterial activity of Ag-TNTS}

Fig.5 shows the antibacterial activity of the coatings. As expected, the pure Ti group (Fig. 5a) was covered by bacterial colonies, indicating that untreated $\mathrm{Ti}$ does not possess antibacterial activity. Compared with pure $\mathrm{Ti}$, the TNTs group (Fig. 5b) inhibited a large number of bacterial colonies, suggesting that TNTs have good antibacterial properties. Similarly, as shown in Fig. 5c, the Ag-TNTs group inhibited significantly more bacterial colonies, indicating that the $\mathrm{Ag}$ ions released from the $\mathrm{Ag}$-TNTs have a strong bactericidal effect

Recently, many scholars have put forward the new concept of integration of structure and biological function on the surface of the implant. The core idea is that the implant supplies its own excellent mechanical properties and corresponding biological functions. Studies have shown that $\mathrm{Ag}, \mathrm{Sr}, \mathrm{Ca}, \mathrm{Si}$ and other elements introduced by surface modification can effectively improve the biological activity of the implant. In view of the broad-spectrum antibacterial activity of $\mathrm{Ag}$ and the excellent structure of our nanotube coating prepared by anodic oxidation technology, we assume that $\mathrm{Ag}$ is introduced onto the surface of the titanium implant and $\mathrm{Ag}^{+}$is slowly released, exhibiting its antibacterial effect ${ }^{22-24}$.

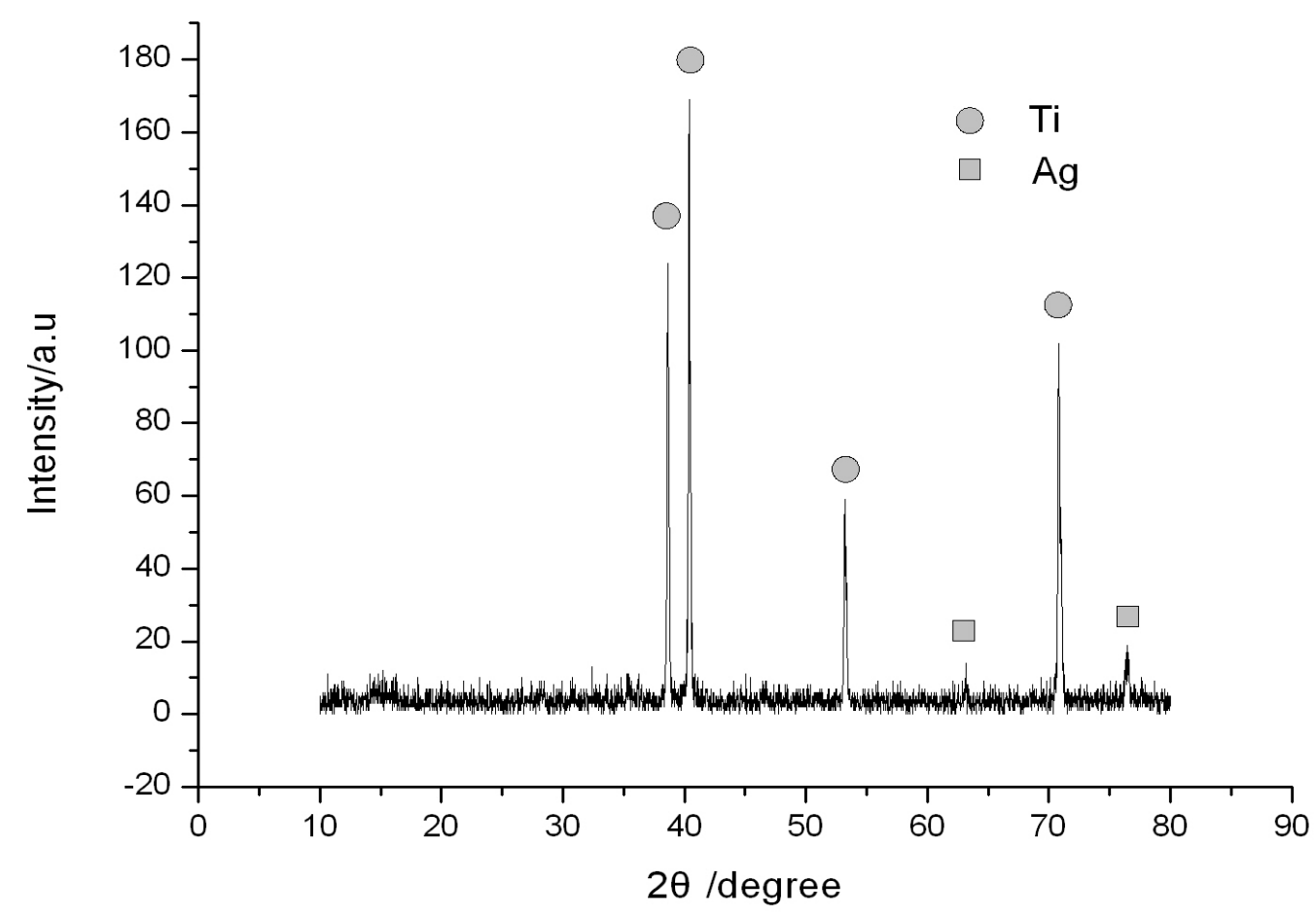

Fig 4. XRD patterns of the Ag-TNT coatings.
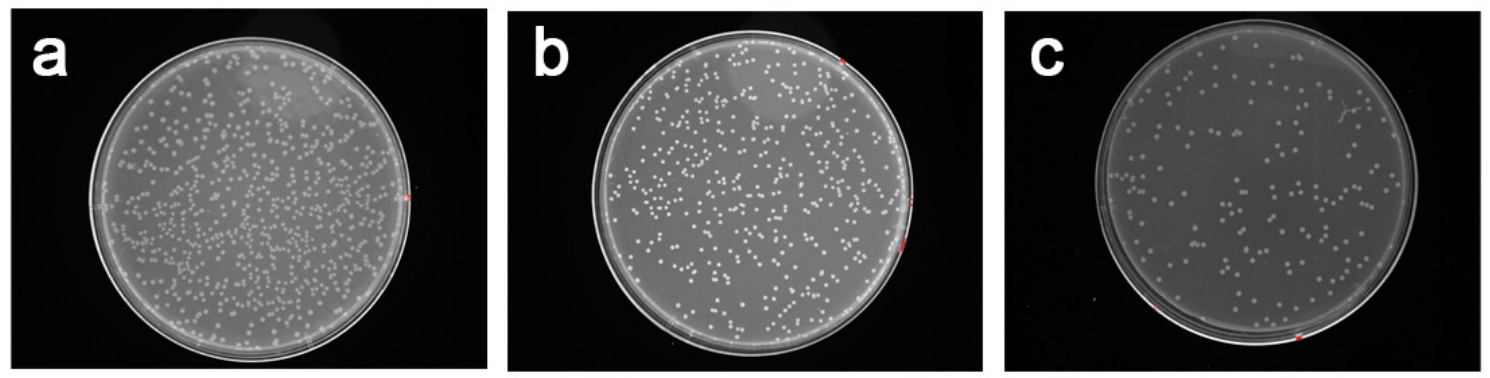

Fig. 5 S. aureus adhering to the Ti (a), TNTs (b) and Ag-TNTs (c). 


\section{Conclusions}

In summary, we have introduced a novel method to fabricate Ag-TNTs coatings on Ti. We first coated TNTs onto the surface of Ti using an anodic oxidation method and subsequently synthesized Ag-TNTs coatings via $\mathrm{AgNO}_{3}$ immersion and xenon light irradiation. Our results showed that Ag was successfully incorporated into TNTs, while our antibacterial experiments showed that the implant exhibited excellent antibacterial activities. The method described is simple and effective. We hope that the unique structure of

\section{References}

1. Geetha M, Singh AK, Asokamani R, Gogia AK. Ti based biomaterials, the ultimate choice for orthopaedic implants: a review. Progress in Materials Science. 2009;54(3):397-425. http://dx.doi.org/10.1016/j.pmatsci.2008.06.004

2. Liu X, Chu PK, Ding C. Surface modification of titanium, titanium alloys, and related materials for biomedical applications. Materials Science and Engineering: R: Reports. 2004;47(3-4): 49-121. http://dx.doi.org/10.1016/j.mser.2004.11.001

3. Darouiche RO. Treatment of infections associated with surgical implants. New England Journal of Medicine. 2004;350:14221429. http://dx.doi.org/ 10.1056/NEJMra035415

4. Wolcott RD, Ehrlich GD. Biofilms and chronic infections. JAMA. 2008;299(22): 2682-2684. http://dx.doi.org/10.1001/ jama.299.22.2682

5. An YH, Friedman RJ. Concise review of mechanisms of bacterial adhesion to biomaterial surfaces. Journal of Biomedical Material Research. 1998;43: 338-348.

6. Zhao L, Chu PK, Zhang Y, Wu Z. Antibacterial coatings on titanium implants. Journal of Biomedical Material Research B: Applied Biomaterials. 2009; 91(1): 470-480.

7. Guo L, Feng W, Liu X, Lin C, Li B, Qiang Y. Sol-gel synthesis of antibacterial Hybrid Coatings on titanium. Materials Letters. 2015;160:448-451. http://dx.doi.org/10.1016/j.matlet.2015.08.027

8. Gao J, Zhao C, Zhou J, Li C, Shao Y, Shi C, et al. Plasma sprayed rutile titania-nanosilver antibacterial coatings. Applied Surface Science. 2015;355: 593-601. http://dx.doi.org/10.1016/j. apsusc.2015.07.147

9. Yang B, Uchida M, Kim H-M, Zhang X, Kokubo T. Preparation of bioactive titanium metal via anodic oxidation treatment. Biomaterials. 2004;25(6): 1003-1010. http://dx.doi.org/10.1016/ S0142-9612(03)00626-4

10. Gong D, Grimes CA, Varghese OK, Hu W, Singh RS, Chen $\mathrm{Z}$, et al. Titanium oxide nanotube arrays prepared by anodic oxidation. Journal of Materials Research. 2001;16(12):33313334. http://dx.doi.org/10.1557/JMR.2001.0457

11. Le Guéhennec L, Soueidan A, Layrolle P, Amouriq Y. Surface treatments of titanium dental implants for rapid osseointegration. Dental Materials. 2007;23(7): 844-854. http://dx.doi.org/10.1016/j. dental.2006.06.025

12. Bai L, Hang R, Gao A, Zhang X, Huang X, Wang Y, et al. Nanostructured titanium-silver coatings with good antibacterial activity and cytocompatibility fabricated by one-step magnetron sputtering. Applied Surface Science. 2015;355: 32-44. http:// dx.doi.org/10.1016/j.apsusc.2015.07.064

13. Arunachalam A, Dhanapandian S, Manoharan C, Sivakumar G. Physical properties of $\mathrm{Zn}$ doped $\mathrm{TiO}_{2}$ thin films with spray pyrolysis technique and its effects in antibacterial activity.
TNTs and antibacterial activities induced by Ag can play a combined role, which have potential significance in medical applications.

\section{Acknowledgements}

This study was supported by the Natural Science Foundation of Jiangsu Province (20140123).

\section{Disclosure statement}

No potential conflict of interest was reported by the authors.

Spectrochim Acta A: Molecular and Biomolecular Spectroscopy. 2015;138:105-112. http://dx.doi.org/10.1016/j.saa.2014.11.016

14. Yao X, Zhang X, Wu H, Tian L, Ma Y, Tang B. Microstructure and antibacterial properties of $\mathrm{Cu}$-doped $\mathrm{TiO}_{2}$ coating on titanium by micro-arc oxidation. Applied Surface Science. 2014;292:944947. http://dx. doi.org/10.1016/j.apsusc.2013.12.083

15. Zhao L, Wang H, Huo K, Cui L, Zhang W, Ni H, et al. Antibacterial nano-structured titania coating incorporated with silver nanoparticles. Biomaterials. 2011;32(24): 5706-5716. http://dx.doi.org/10.1016/j.biomaterials.2011.04.040

16. Zheng Y, Li J, Liu X, Sun J. Antimicrobial and osteogenic effect of Ag-implanted titanium with a nanostructured surface. International Journal of Nanomedicine. 2012;7:875-884. http:// dx.doi.org/10.2147/IJN.S28450

17. Macak JM, Tsuchiya H, Schmuki P. High-aspect-ratio $\mathrm{TiO}_{2}$ nanotubes by anodization of titanium. Angewandte Chemie International Edition. 2005;44:2100-2102. http://dx.doi. org/10.1002/anie.200462459

18. Wang N, Li H, Lü W, Li J, Wang J, Zhang Z, et al. Effects of $\mathrm{TiO} 2$ nanotubes with different diameters on gene expression and osseointegration of implants in minipigs. Biomaterials. 2011;32(29):6900-6911. http://dx.doi.org/10.1016/j. biomaterials.2011.06.023

19. Hacking SA, Boyraz P, Powers BM, Sen-Gupta E, Kucharski W, Brown CA, et al. Surface roughness enhances the osseointegration of titanium headposts in non-human primates. Journal of Neuroscience Methods. 2012;211(2):237-244. http://dx.doi. org/10.1016/j.jneumeth.2012.09.002

20. Feng QL, Wu J, Chen G.Q, Cui FZ, Kim TN, Kim JO. A mechanistic study of the antibacterial effect of silver ions on Escherichia coli and Staphylococcus aureus. Journal of Biomedical Material Research. 2000;52(4):662-668.

21. Greulich C, Braun D, Peetsch A, Diendorf J, Siebers B, Epple $\mathrm{M}$, et al. The toxic effect of silver ions and silver nanoparticles towards bacteria and human cells occurs in the same concentration range. RSC Advances. 2012;2(17):6981-6987. http://dx.doi. org/10.1039/C2RA20684F

22. Surmenev RA, Surmeneva MA, Ivanova AA. Significance of calcium phosphate coatings for the enhancement of new bone osteogenesis - A review. Acta Biomaterialia. 2014;10(2):557579. http://dx.doi.org/10.1016/j.actbio.2013.10.036

23. Zhang Z, Sun J, Hu H, Wang Q, Liu X. Osteoblast-like cell adhesion on porous silicon-incorporated $\mathrm{TiO}_{2}$ coating prepared by micro-arc oxidation. Journal of Biomedical Material Research B : Applied Biomaterials. 2011;97(2):224-234.

24. Kung K-C, Lee T-M, Lui T-S. Bioactivity and corrosion properties of novel coatings containing strontium by micro-arc oxidation. Journal of Alloys and Compounds. 2010;508(2):384-390. http:// dx.doi.org/10.1016/j.jallcom.2010.08.057 